\title{
Building models of territorial distributed systems
}

\author{
Gulbaev Nematilla Abdukarimovich ${ }^{1}$, Khalmetova Mukhabbat Khudainazarovna², \\ Sobirova Saida Ravshabekovna ${ }^{3}$ \\ ${ }^{1}$ Associate Professor, Candidate of Technical Sciences \\ ${ }^{2}$ Lecturer, Chirchik State Pedagogical Institute, Tashkent Region \\ ${ }^{3}$ teacher of the 18th secondary school in Chirchik
}

Email: gulbaev_n@umail.uz

\begin{abstract}
With the help of a formal-logical approach, the interrelationships of organizational and functional elements of management structures are determined, the concept of a task is also given from the standpoint of the theory of sets and relations.
\end{abstract}

Keywords: model, system, functional elements, management structures.

\section{INTRODUCTION}

In recent years, the literature has covered. On the issues of the structure, implementation and application of control systems for distributed information processing, an overview of the advantages that distributed processing provides in comparison with the centralized use of data and computing power is given.

In [1,2], the issues of effective use of information processing tools by many users are considered, the problems of managing these tools and a network of devices are solved.

In electrical networks, the problems of control reliability are solved not by means of data processing, but by dispersed, diverse network objects (power lines, substations, switchgears, etc.), problems of ensuring the operation mode of network equipment interconnected by material flows, control and analysis of the technical condition of objects dispersed over large areas, as well as control of processing, reception and transmission of data of dispersed objects on hierarchical levels of management. Automated control systems of power systems are being developed as part of an automated dispatch control system (ASDU) of power systems and power grids. In these works, network control at the PES level and inside the substation technological control is practically not considered. The works $[2,3]$ investigate the issues of operation of electrical networks, operation schemes and methods for determining the economic efficiency of the automated control system of the PES. Possible approaches to solving the problems of planning the number of power grid personnel are considered in [1]. In [1,2], the improvement of the operation of electrical networks of $0.4-20 \mathrm{kV}$ in rural areas is considered, the damageability of electrical networks and their elements is analyzed, organizational and technical measures aimed at ensuring the reliability of power supply to rural networks are determined. Modern methods of organizing and carrying out repair work, technical and operational maintenance, issues of managing the operation of electrical networks, forms of work with personnel and improving the NU qualifications are described in works [3].

The study showed that the problem of system analysis of network management, the synthesis of control systems and the construction of models of geographically distributed systems are poorly studied. Therefore, it becomes necessary to develop the following scientific and practical problems in the field of geographically distributed systems:

- development of methods for building a management model; system analysis and synthesis of management;

- development of algorithms and software for control problems.

The formulated problems should be solved taking into account the practical results obtained in the creation and operation of existing control systems for electrical networks, as well as using the methods used for the mathematical description of geographically distributed systems. The use of statistical methods meets a number of difficulties. Therefore, it is convenient to solve the problem from the standpoint of a formal-logical approach based on the elements of the theory of relations [1]. In this case, the description of geographically distributed systems is limited by relations, each of which is built on a pair of sets of elements of this system.

In the studies considered, individual problems of control of electrical networks were solved, but their holistic analysis from the systemic positions of control is absent. A systematic approach to the development of control problems, in which the system is considered as consisting of a large number of subsystems and tasks, is most suitable for solving the problems of improving the structure of control systems for electrical networks. Indeed, the integrated description makes it possible to highlight the general imbalances in the distribution of the number of management personnel of the PES and explicitly answers questions; where and what kind of inconsistencies and tensions take place in the analyzed system, identifies general specific management functions, recommends complex methods of planning and implementing measures for long-term, current and operational management.

Enterprises of electrical networks are the most widespread class of objects in distributed systems. Indeed, the unified energy system (UES) of the country covers more than 60 energy systems. Moreover, the total number of TPPs in them is more than a thousand. They provide electricity to all industrial, agricultural and other objects of the national 
economy. Their importance in the management of the national economy is enormous, even a short-term disruption of the functioning of electrical networks can lead to irreparable losses of industrial, agricultural and other products.

In order to clarify the current state of problems in the management of electrical networks, we will consider the results obtained by us during the survey of electrical networks of the Republic of Uzbekistan, the Republic of Azerbaijan, Volgograd, Tomsk, Odessa regions.

The study of control systems for electric grids was carried out by methods of direct, selective, questionnaire surveys, interviewing performers at workplaces, personal participation in work, obtaining information through conversations and consultations.

They determined all the documentation, the principles of its formation, established where and how the information used in the document was obtained, found out the methods of forming schemes of document flows, performers, the address of receipt of documents, kept a record of all planned, reporting and mandatory documentation (orders, orders, acts, invoices, operational defective statements, etc.), documentation related to the correction of previously transmitted messages or changes to this information, reference information on this document, functions performed by individual departments, performers, etc.

For each task, the input and output information, performers and the frequency of solving tasks, the address of receipt of the results of the task, the complexity of the solution and the relationship with other tasks were determined.

1. Building a model for analyzing the management structure, which includes:

- $\quad$ building models of objects distributed throughout the enterprise

$$
\begin{aligned}
M_{B}=\left\langle I_{B} ; R_{1}, \ldots, R_{m}>;\right. \\
\text { Region } \quad \begin{aligned}
M_{h} & =<I_{h} ; R_{1}, \ldots, R_{m}>; h=(1, H) \overline{;} \\
M_{y} & =<I_{y} ; R_{1}, \ldots, R_{m}>; y=\overline{1, y_{h}} ;
\end{aligned}
\end{aligned}
$$

Plot

where $\boldsymbol{I}_{\boldsymbol{B}}, \boldsymbol{I}_{\boldsymbol{h}}, \boldsymbol{I}_{\boldsymbol{y}}$ - sets of objects;

- construction of models of subsystems determined by the objective functions $F$ :

$$
M_{F}=\left\langle S(F), R_{1}, \ldots, R_{m}\right\rangle ;
$$

where $\boldsymbol{S}(\boldsymbol{F})$ is a set of subsystems determined by target control functions; building problem models

$$
M_{D}=\left\langle D ; R_{1}, \ldots, R_{m}>;\right.
$$

where $\boldsymbol{D}$ is a set of tasks for managing sets of objects $\boldsymbol{I}_{\boldsymbol{B}}, \boldsymbol{I}_{\boldsymbol{h}}$ and $\boldsymbol{I}_{\boldsymbol{y}}$;

ordering of structure elements

$$
\begin{aligned}
& \mathbf{M}_{\mathbf{B}}=\left\langle\mathbf{I}_{\mathbf{B}} ; \mathbf{R}\right\rangle ; \quad \mathbf{M}_{\mathbf{h}}=\left\langle\mathbf{I}_{\mathbf{h}} ; \mathbf{R}\right\rangle ; \quad \mathbf{M}_{\mathbf{y}}=\left\langle\mathbf{I}_{\mathbf{y}} ; \mathbf{R}\right\rangle ; \\
& \mathbf{M}_{\mathrm{F}}=\langle\mathrm{S}(\mathbf{F}), \mathbf{R}\rangle ; \quad \mathbf{M}_{\mathbf{D}}=\langle\mathbf{D} ; \mathbf{R}\rangle,
\end{aligned}
$$

where $\boldsymbol{R}$ is the preference relation for the elements of the sets;

- grouping of structural elements according to their relationships;

- determination of the number of hierarchical levels of management $\mathbf{L}$, the degree of centralization $\boldsymbol{\alpha}_{\mathrm{i}}=\frac{\boldsymbol{w}_{\boldsymbol{i}}}{\boldsymbol{w}_{\boldsymbol{i}-1}}$, where $W_{i}, W_{i-1}$ - are the set of tasks to be solved at the $\mathbf{l}$ - and (l-1) levels of management $(\mathbf{l}=\overline{\mathbf{1}, \boldsymbol{L}}), \boldsymbol{\lambda}_{\mathrm{ij}}-$ the degree of specialization of the enterprise divisions;

- determining the economic efficiency of ordering structural elements.

2. Building a model for the synthesis of a control structure, which consists in finding: [2].

- distribution of a set of objects on hierarchical levels of management

$$
\psi_{I: I_{B} \rightarrow L}
$$

distribution of tasks $\boldsymbol{D}$ by functional subsystems

$$
\psi_{s: D}: S(F)
$$

the number of control subsystems $\boldsymbol{S}_{\boldsymbol{y}}$;

- distribution of the set of functions over the control subsystems

$$
\psi_{\text {s: }} \boldsymbol{F} \rightarrow \boldsymbol{S}_{y: \text {. }}
$$

3. Development of a management model, which includes:

- $\quad$ building a model of the state of objects distributed on the territory enterprises 
district

$$
M_{Q_{\mathrm{B}}}=<I_{B T}(Q) ; R_{1}, \ldots, R_{m}>;
$$

plot

$$
M_{Q_{h}}=<I_{h T}(Q) ; R_{1}, \ldots, R_{m}>
$$

$$
M_{Q_{y}}=<I_{y T}(Q) ; R_{1}, \ldots, R_{m}>;
$$

where $I_{\boldsymbol{B} \boldsymbol{T}}(\boldsymbol{Q}), \boldsymbol{I}_{\boldsymbol{h} \boldsymbol{T}}(\boldsymbol{Q}), \boldsymbol{I}_{\boldsymbol{y} \boldsymbol{T}}(\boldsymbol{Q})$ - a set of objects with sets of states

$Q=\left\{q_{1}, q_{2}, \ldots, q_{h}\right\}$

determination of the correspondence of objects and their set of states at the moment of time $\mathbf{T}$

$$
\boldsymbol{\psi}_{Q_{\mathrm{BT}}}: \boldsymbol{I}_{B T} \quad \boldsymbol{Q}_{B T},
$$

where $\boldsymbol{L}_{\mathbf{B T}}, \mathbf{Q}_{\boldsymbol{B}}$ - a set of objects and their states;

- building a model of current control, which consists in determining

$$
M_{T}=\left\langle I_{B T}\left(Q_{T}\right) \cdot J_{B T}\left(E_{B T}\right), E_{B T}, A_{B T} ; R_{1}, \ldots, R_{m}>,\right.
$$

where $\boldsymbol{M}_{T}$ is the current management model; $\boldsymbol{I}_{\boldsymbol{B} T}\left(\boldsymbol{Q}_{T}\right)$ - a set of objects, the state of which at the moment $T$ is characterized by $\boldsymbol{Q}_{T} ; \boldsymbol{J}_{\boldsymbol{B} T}\left(\boldsymbol{E}_{\boldsymbol{B} T}\right)$ - a set of works due to the presence of many $\boldsymbol{E}_{\boldsymbol{B} T}$ defects; $\boldsymbol{A}_{\boldsymbol{V} \boldsymbol{T}}$ - a set of material, monetary, labor resources;

conformity of works and defects

$$
\psi_{E_{\mathrm{BT}}}: E_{B T} \rightarrow J_{B T}\left(E_{B T}\right)
$$

correspondence of defects and states of objects

$$
\psi_{Q_{\mathrm{ET}}}: E_{B T} \rightarrow Q_{B T} ;
$$

conformity of works and objects

$$
\psi_{J_{\mathrm{BT}}}: J_{B T}\left(E_{B T}\right) \rightarrow I_{B T} Q_{B T} ;
$$

building a model of operational management, which consists in determining

$\boldsymbol{M}_{\tau}=\left\langle\boldsymbol{I}_{\boldsymbol{B} \tau}\left(\boldsymbol{E}_{B}\right), \boldsymbol{J}_{\boldsymbol{B} \tau}\left(\boldsymbol{E}_{B \tau}\right) ; \boldsymbol{E}_{B \tau}, \boldsymbol{A}_{\boldsymbol{B} \tau}, \boldsymbol{U}_{B \tau} ; \boldsymbol{R}_{1}, \ldots, \boldsymbol{R}_{m}>\right.$, Where $\boldsymbol{M}_{\tau}$ - is the operational management model; $\boldsymbol{И}_{\boldsymbol{B} \tau}$ - many performers;

distribution of work by performer

distribution of work in time

$$
\psi_{H \pi}: J_{B x}\left(E_{B x}\right) \rightarrow H_{B \pi}
$$

$$
\psi_{t}: J_{B x}\left(E_{B x}\right) \rightarrow T \text {. }
$$

4. Development of an operator control model, which consists in determining: models of transmitting system nodes

$$
Y_{i}=q_{i} X_{i}+r_{i}, \quad i=\overline{1 ; h}
$$

where $\boldsymbol{q}_{\boldsymbol{i}}$-is the node transfer function; $\boldsymbol{X}_{\boldsymbol{i}}$ - signal at the input of the i-th node; $\boldsymbol{Y}_{\boldsymbol{i}}$ - output signal; $\boldsymbol{r}_{\boldsymbol{i}}$ is the probability of transmitting a false signal;

- models of systems for connecting operators serving distributed objects;

- number of operators.

5. Development of a control reliability model, including [3]:

- building a model of data transmission from distributed objects;

- determination of the performance measure of distributed objects;

- $\quad$ structural methods to improve the reliability of the functioning of dispersed objects;

- operational methods for improving reliability; determination of the effective range of distributed objects taking into account reliability.

Unlike other methods of constructing models, the formal-logical one takes into account the peculiarities of geographically distributed systems. The problems of reliability of the control line for hierarchical levels are currently considered mainly for technical systems, and for the class of a territorially distributed system of electrical networks, it has not yet been practically solved. 


\section{REFERENCES}

1. E. Paiziev, N.A. Gulbaev. Models of systems with dispersed objects. Tashkent: Fan. 1984.

2. N.A. Gulbaev., N.A. Kudratilloev. STATUS OF MANAGEMENT PROBLEMS IN SYSTEMS WITH DISPERSED OBJECTS (ON THE EXAMPLE OF ELECTRIC NETWORKS). (STATE OF CONTROL PROBLEMS OF SYSTEMS WITH REDUCED OBJECTS (ON THE EXAMPLE OF ELECTRIC NETWORKS)).

3. SCIENCE AND WORLD International scientific journal № 6 (82), 2020, Vol. II

4. Gulbaev N.A., Duisenov N.E., Akhmedov B.A., Rakhmanova G. S. Models of electrical network management systems Models of electric network management systems. "Young Scienist \# 22 (312) - May 2020. 